\title{
Analysis of Sub-processes Specific to Hybrid Non-conventional Machining Methods
}

\author{
Hans-Peter Schulze and Oliver Kröning
}

Hans-Peter Schulze. Leukhardt Schaltanlagen Systemtechnik GmbH NL Magdeburg, Gustav-Ricker-Straße 62, 39120 Magdeburg, Germany

Corresponding author : hans-peter.schulze@leukhardt-system.de

Oliver Kröning. Leukhardt Schaltanlagen Systemtechnik GmbH NL Magdeburg, Gustav-Ricker-Straße 62, 39120 Magdeburg, Germany

\begin{abstract}
In principle, all non-conventional machining processes can be viewed as the sum of various subprocesses, the separate analysis of which influences the effectiveness of this machining process. The first question to answer is the criteria by which these sub-processes can analyzed. Since the non-conventional processes work exclusively based on electrical energy, voltage and current determine the character of the sub-process. The second focus is the time constant with which voltage and current influence the process, from which the measurement of the corresponding parameters result. The third group of influencing parameters is the working medium and its change due to the main removal process. In the summary, these three groups are consider with regard to a process model with which a process control (technology), a process regulation (process stability) and a safety control (limit value exceeded) are guide.
\end{abstract}

Keywords. Non-conventional Machining, EDM, ECM, Hybrid Processes, Process Analysis

\section{Introduction}

It can be assumed that all non-conventional, hybrid-machining processes consist of a limited number of sub-processes, and that their specifics are only changed by the boundary conditions [1]. A correct process analysis of the sub-processes can thus determine which removal mechanisms, wear rate, and surface topologies are to be expected. Knowledge of the process energy source (PES) and the chemical-physical properties of the working medium can then be used to set the desired process properties and technological parameters via the process parameters. The process analysis is mainly possible due to the current and voltage curves directly at the working gap, whereby the main problem is the measurement of these electrical quantities. Since it is usually not possible to measure the gap voltage, the "real" gap voltage must be determined using an equivalent circuit diagram and the measurement of the various impedances that occur. The article deals with the individual sub-processes and names the boundary conditions that lead to the transition between the sub-processes.

\section{Basic sub-processes and their current and voltage curves}

The basic sub-processes are spark erosion (EDM) and electrochemical removal (ECM), which essentially depend on which working medium is used. In Fig. 1 it is shown in which areas of electrical conductivity these basic processes take place and where the transitions to the hybrid erosion effects occur. The basic EDM process is determined by the working current (Fig. 1, a), which is determined by the impedance of the supply line and contact (connection impedance) in the case of the most commonly used voltage sources (PES). Current sources can be used modularly as a power unit, whereby it must be noted that the voltage changes shift. The ignition voltage (Fig. 1, c) is specified in most applications, but can be lower due to the process or only take place after a time delay. In the case of separate modules of the ignition and power unit, it must be ensured that the currents that are established are specified by the respective unit and are different. The advantage of the modular structure is the significantly lower power consumption. The switching peak of the ignition current is significantly reduced (Fig.1, c), but the switching current peaks can be larger. 
Another very important parameter is the spark burning voltage (Fig. 1, e), which depends on the material pairing (cathode-anode). If a high-frequency oscillation is superimposed on it, one is dealing with a spark discharge, while a smooth burning voltage suggests an arc.

The second basic process is the ECM, which in principle has fewer influencing factors than the EDM.

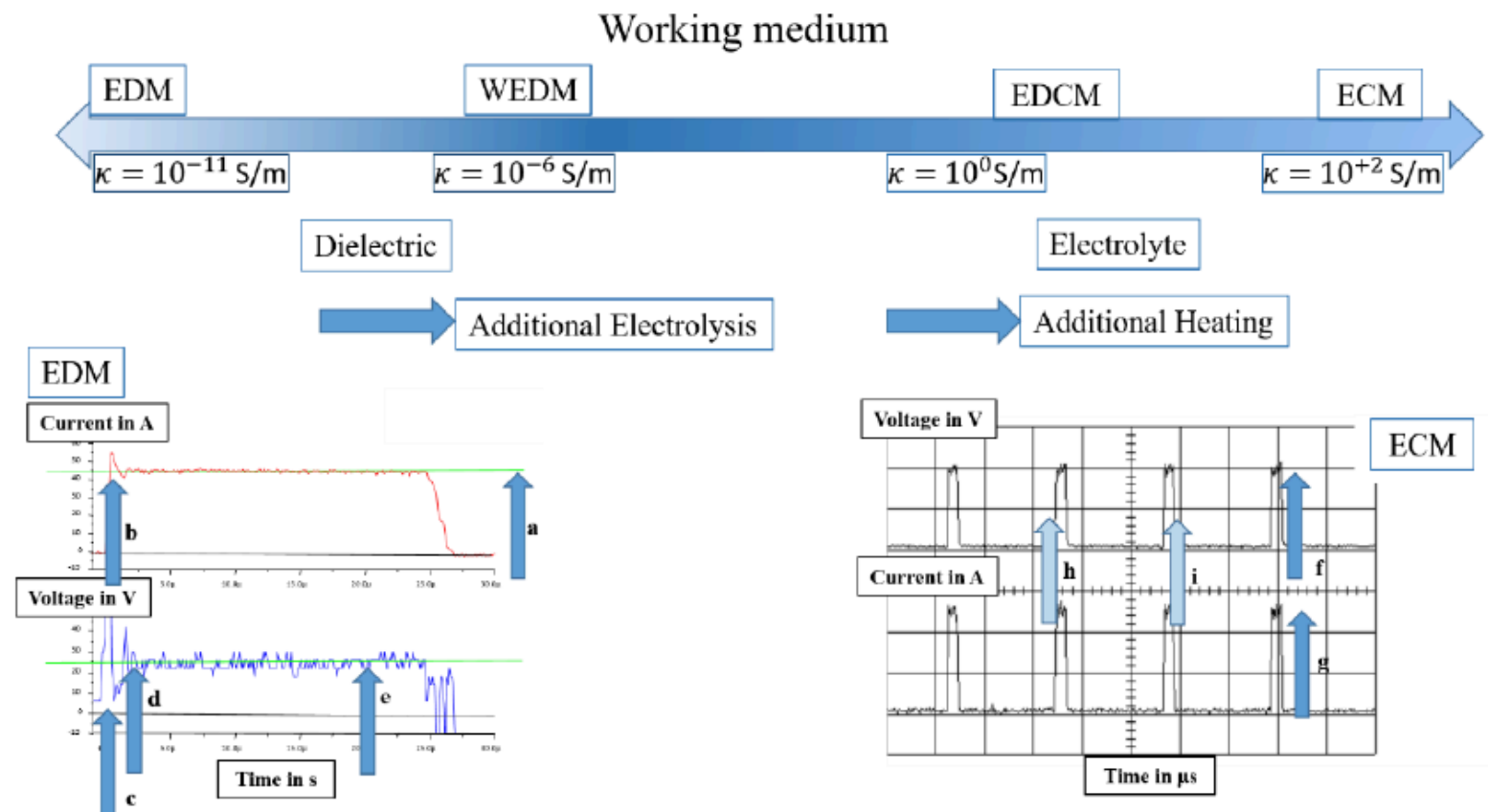

Fig. 1. Basis-Subsystems depending on the conductivity of working medium and they are characteristically $\mathbf{u}$ - and icurves.

If the ECM is also operated with a DC source, the gap voltage (Fig. 1, f) or the working current (Fig. 1, g) are constant, with the current being reduced by changing the gap conditions for a voltage source. For the pulsed ECM (PECM), the connection impedances are of course decisive, which working current is established when a voltage source is used as PES. In this case, two additional modules can supplement the PES. When the gap voltage is switched on, a rise time unit (RTU) can be used, whereby the current rise is increased several times [2] (Fig. 1, h). This RTU is necessary if very short pulses $(<5 \mu \mathrm{s})$ and very high working currents $(>200 \mathrm{~A})$ are to be implemented $[3,4]$. A negative pulse at the end of the EC pulse leads to rapid depassivation and reaching zero gap voltage (Fig.1, i). 


\section{Sub-Processes in the group of thermal processes}

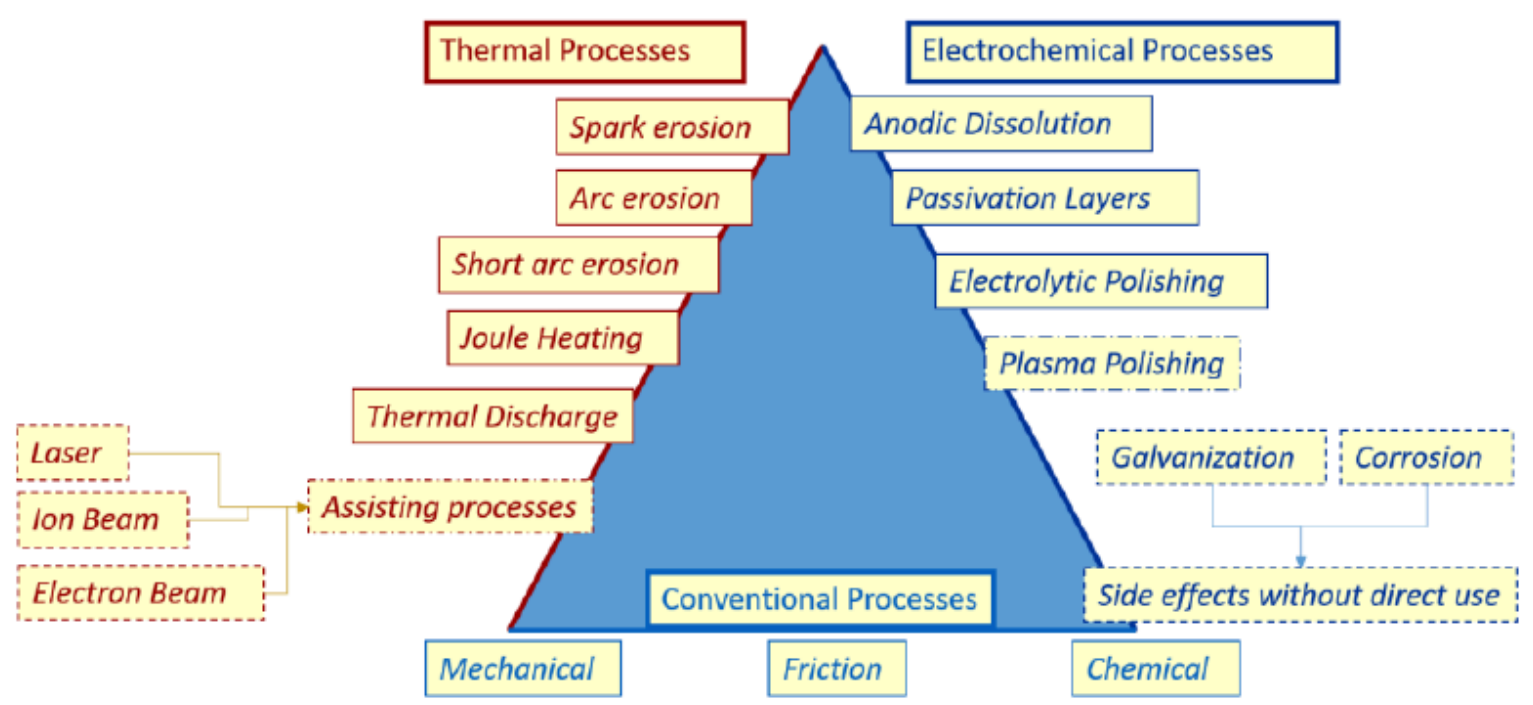

Fig. 2. Sub-processes on the basic processes spark erosion and ECM.

In Fig. 2 it is shown how the classification of the various sub-processes can be made. The top of the thermal processes is spark erosion and top of electrochemical process is anodic metal dissolution. A burning voltage that is different from the spark burning voltage characterizes the arc discharge. The arc current also deviates from the spark current and, as already mentioned, has a smooth current curve. When measuring these process variables, it must be taken into account to what extent the connection inductance has smoothed this value. The advantage of the arc discharge is the higher thermal removal rate, but this can also lead to more critical surface morphologies. With the short arcs, there is a further subdivision that can be analyzed using the current and voltage curves. In the case of short arcs, the focal points (arc break) are critical because they have deep thermal effects.

In the case of Joule heating, a distinction must be made between Joule heating on / in the electrodes and heating of the working medium. The first form has an influence on the impedance of the supply line, which changes the electrical voltage ratios and capacitive switching behavior of current and voltage. The most critical case of Joule heating occurs in the event of a short circuit, which must be excluded for the two basic processes because the workpiece and / or the tool can be destroyed. For the combinations with rotating tool electrodes, the mechanical-thermal removal of the short circuit can be permitted if the temporal effect is in the $\mu$ s range and the short circuit resolves itself.

A final sub-process is the thermal discharge, which takes place primarily when the working medium reaches a sufficiently high electrical conductivity. The thermal effect and the applied electrical field strength create an erosive plasma channel, with the burning voltage, the burning current and the current increases differing from the basic process. The voltage rises are significantly flatter and the plasma channel formation slower but more voluminous. The sub-processes of the thermal processes can supplemented by assisting processes such as Laser, EBM or IBM (Fig. 2).

\section{Sub-processes in the group of electro-chemical processes}

These processes can essentially be analyzed through the voltage and current ranges, the properties of the working medium and the gap width. Passivation represent an extensive impairment of the ablation mechanisms, which can be controlled by knowing the current density value. Dissolution or thermal destruction of the passive layer then come into question for the hybrid processes. 
The occurrence of polishing effects can be increased by the choice of the working medium, whereby it can only be implemented as a sub-process under pulsed conditions with changing current and voltage conditions and controlled gap setting. Since the ECM process usually takes place without wear on the cathode, it must be ensured that no galvanic processes take place on the cathode. The edge effect of corrosion is a non-controllable influence with very long machining times or with insufficient cleaning of the workpiece after machining. In the process, of course, surface oxidation can be classified as corrosion.

Plasma polishing effects arise when a plasma layer influences the surface processing. However, this can hardly occur in combination with other subsystems apart from EC polishing, so that separate voltage-current characteristics apply here.

\section{Hybrid processes and their analysis}

\subsection{Wire-EDM}

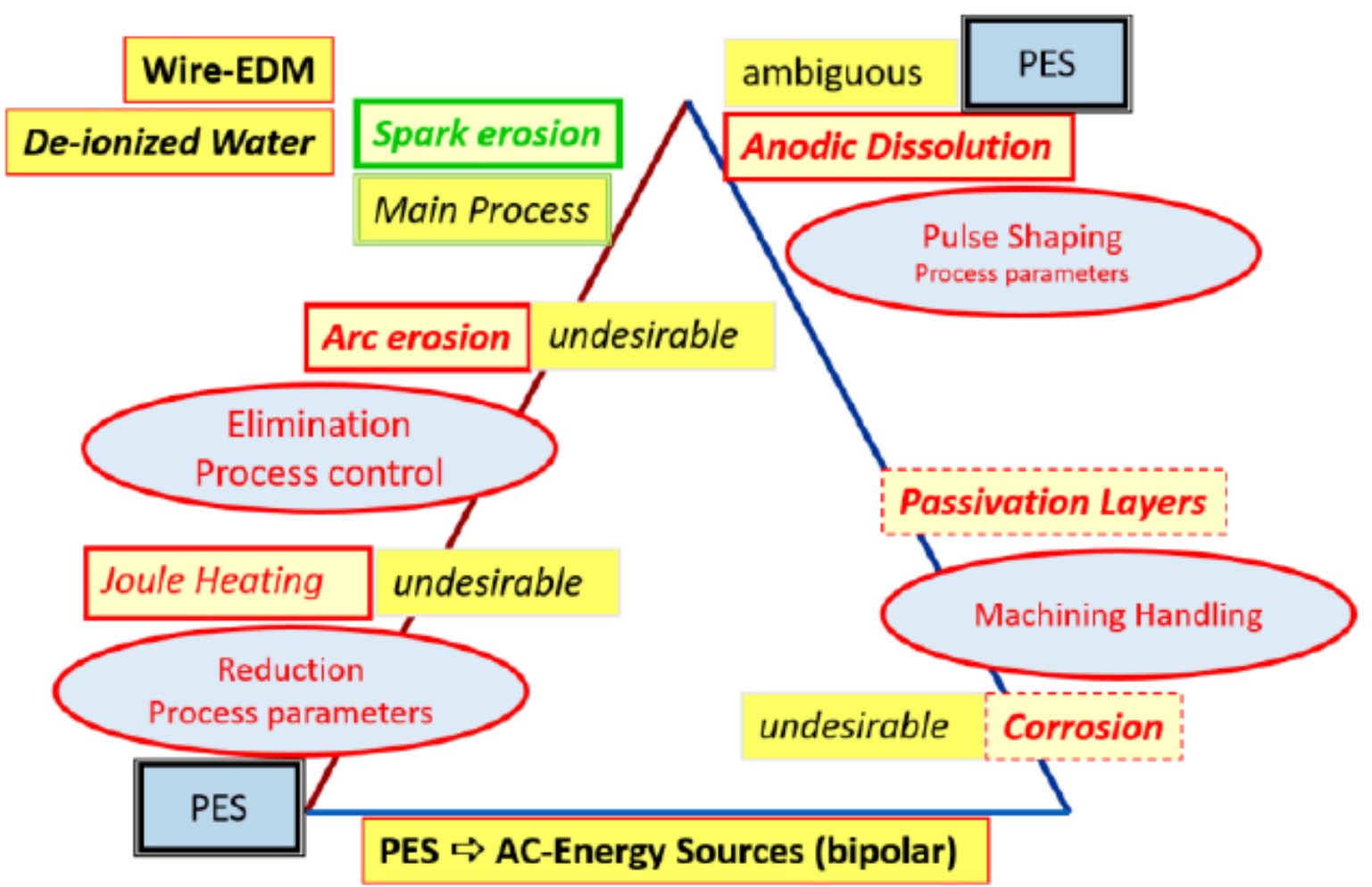

Fig. 3. Sub-processes by WEDM.

In the case of hybrid processing methods, the sub-processes can be divided into the desired assisting processes and the random processes. The latter cannot necessarily be found in the current and voltage curves, or no clear parameter definition can be made for them. This factor occurs, for example, in WEDM with de-ionized water. The electrolytic side effects are very often undesirable and can only be reduce by a specific selection of the pulse parameters (Fig. 3). Arc discharges and very high Joule heating can lead to wire breaks, which must be prevented. In the case of Joule heat, the process parameters can be reduced. In the case of arcs, a prediction must be made, which cannot be avoided by the process parameters but by the process regulation. 


\subsection{High-Speed Wire-EDM}

For the high-speed wire EDM [5], a sufficient process analysis can be carried out according to Fig. 4. Basically, five types of pulses can be identified, which can be assigned to very specific sub-processes. Type 1 in Fig. 4 is a spark discharge without ignition delay, which corresponds to local thermal erosion. Type 2 in Fig. 4 is a thermal breakdown, which can be seen from the fact that a phase of heating takes place before the actual breakdown. With a current close to zero, it would be an ignition delay of Type 1 .

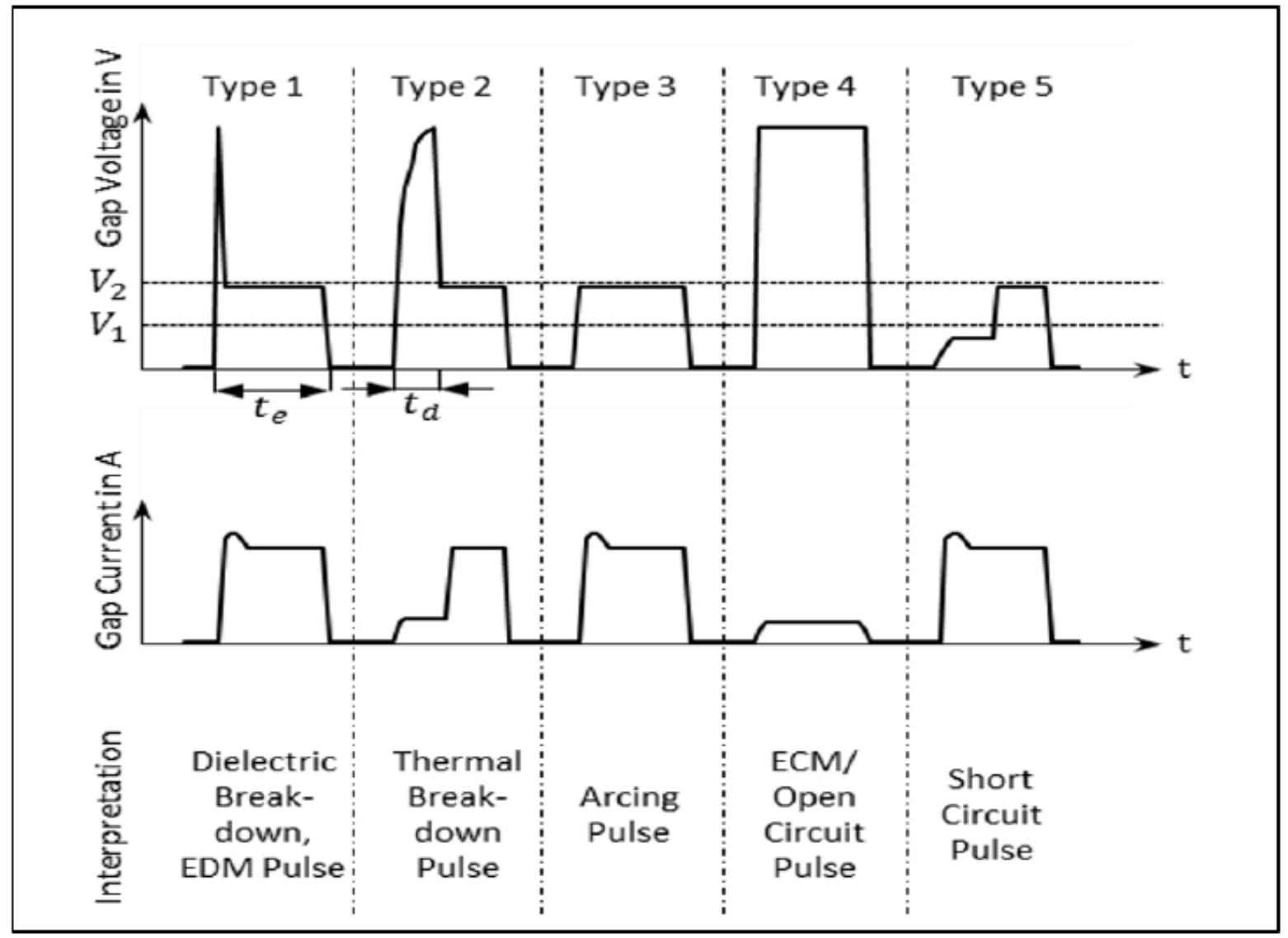

Fig. 4. Schematic selection of working pulses for HS-WEDM [5]

With Type 3, an arc can be analyzed, whereby the operating voltage is usually slightly below the voltage V2 specified here. The arc places a higher thermal load on the surface and can lead to severe metallurgical damage in the surface if the base point is torn off. Type 4 are generally EC pulses, with no-loads looking similar but having a much lower current. The EC currents are higher and correspond to the heating level of Type 2.

Type 5 shows a short circuit, where the voltage curve shows that this short circuit dissolves during the pulse. Since the voltage drops then first show a voltage close to, zero, whereby the selection criterion should be slightly lower than the arc voltage. The subsequent voltage increase to the spark voltage indicates discharges from the short circuit. The currents then correspond to Type 1 or Type 3. The displayed low voltage is due to the fact that the measured voltage does not equal zero because the measuring contacts are located away from the working gap and therefore have a low resistance.

The changed thermal breakdowns with different electrical conductivities of the working medium can be seen in FIG. At $1 \mu \mathrm{S} / \mathrm{cm}$ the electrical breakdown takes place, as it is known from EDM. There is a clear ignition delay without 
any current component. If the electrical conductivity is 500 times higher, a current of 1 - 2 A must be measured for the voltage delay. The voltage of the breakdown drops slightly and a very brief re-ignition can be seen in the area where the pulse is switched off. The current amplitude is also slightly below that of the spark discharge. The further sevenfold increase in the conductivity roughly doubles the initial current, which suggests that the plasma channel is enlarged or that the gap width changes. For the next doubling of the initial current, only a tripling of the conductivity is necessary. Overall, the electrical conductivities of the working medium are still well (10 times) below the usual values for the ECM.

\subsection{Electric-discharge-electro-chemical machining (ELESIN)}

Another hybrid process is EDCM (ELESIN) [6] in which an electrochemical ablation with a passivating electrolyte occurs in the assisting process. The passivation, which reduces the EC erosion, is destroyed by electrical discharges (main process), which makes ECM possible again. The spark breakdown differs from the EDM process in that it occurs in the dielectric passivation layer.

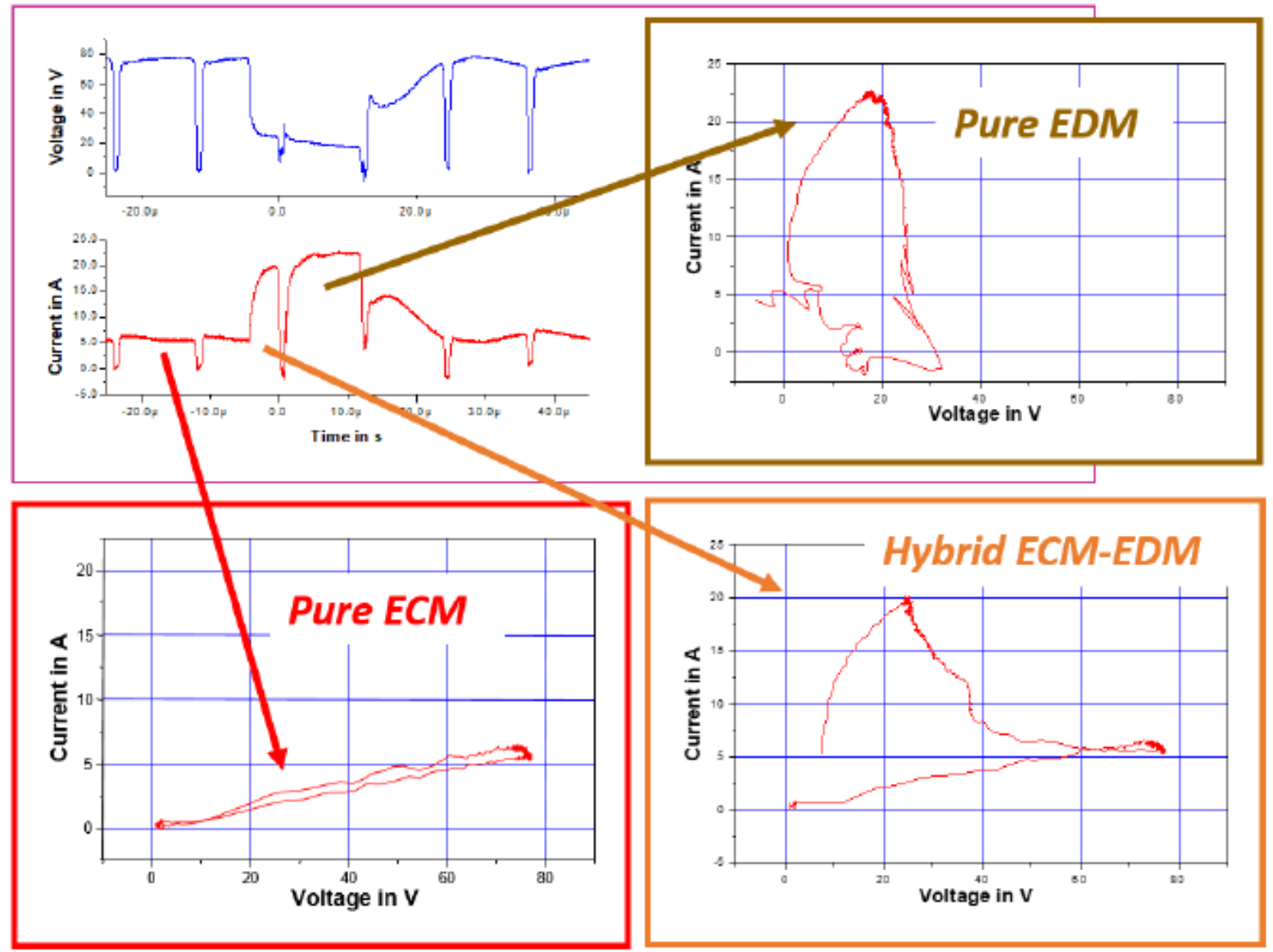

Fig. 6. Voltage and current curves for ELESIN and the characteristic u-i-Diagrams for the Sub-Processes [6].

The electrical voltage limitation means that the lateral working gap remains constant and is passivated after a certain processing time. In the front working gap, the feed speed causes a constant change between EDM and ECM. Fig. 6 shows how the associated current-voltage characteristics look and how the time courses change. 


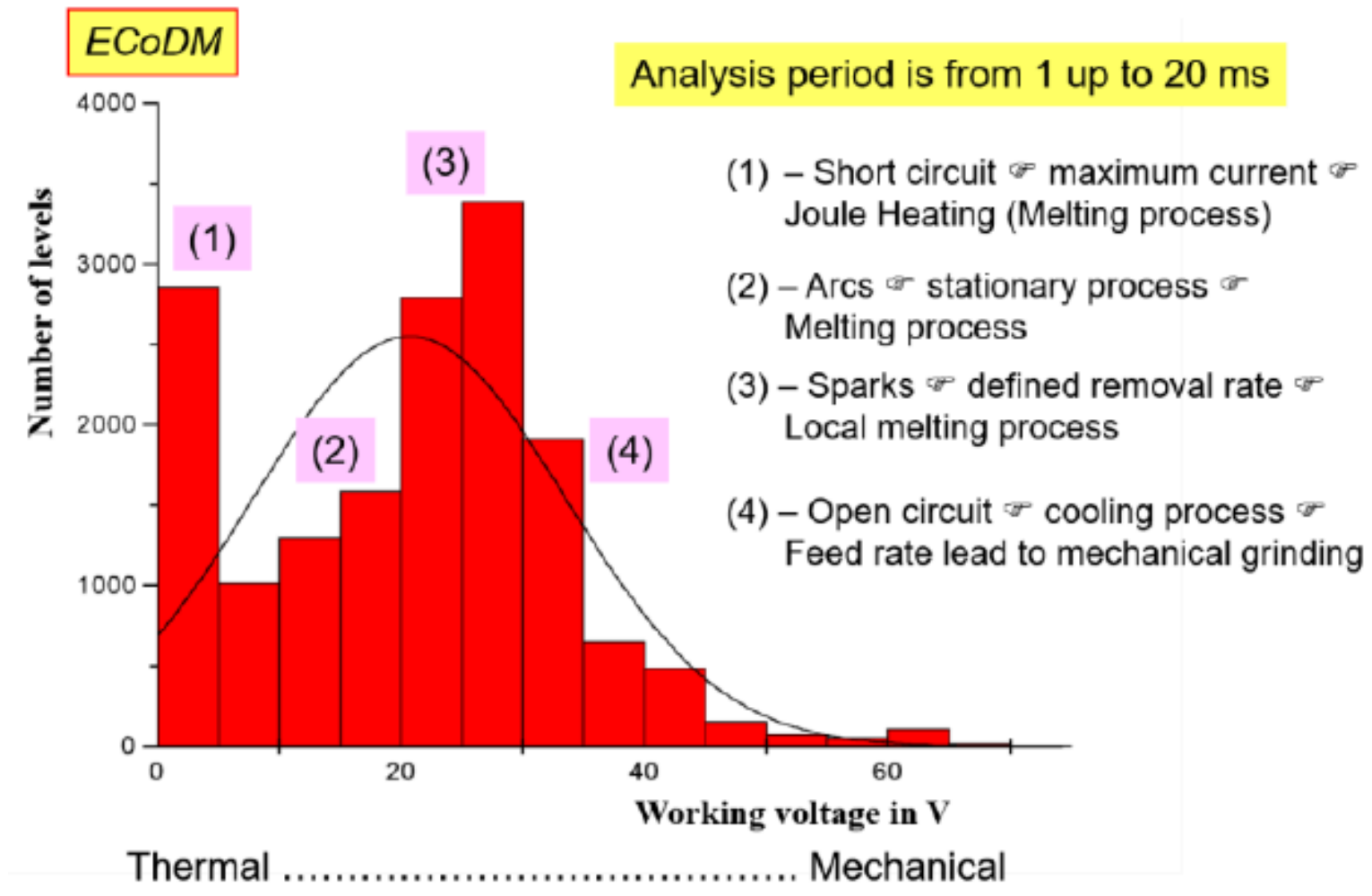

Fig. 7. Voltage and current curves for ECoDM and their u-characteristics [7].

A final classification is possible if the sub-processes result from constantly changing voltage values (e.g. sine function). In this case, the current and voltage must be analyzed according to their frequency distribution. With ECoDM with a rotating disc and gaseous working medium [1], it is not possible to influence each individual sub-process, but only the primary sub-processes within an analysis period

Fig. 7 shows a distribution function (histogram) for the machining voltage with the assignments to the individual sub-processes. For this application, we have a high number of spark discharges and thermal removal rates from short circuits. The arc erosion is reduced. When cutting steel cables (strands), the result of the cut is to be expected to fuse the strands $[1,7]$.

\section{Conclusions}

The sub-processes of a hybrid machining process can defined very precisely by measuring the voltage and current at the working gap and thus assigned to the removal process or tool wear.

The requirements for a correct analysis are knowledge of the process energy source (PES), the measuring principle and the measuring accuracy of current and voltage, the equivalent circuit diagram of the hybrid process for measured value correction and the structural influences of the individual sub-processes.

The results of this sub-process analysis are improved process regulation of the feed rate and improved optimization (process control) of the technological process parameters. 
The critical sub-processes that correspond to their physical behavior and thus their current-voltage characteristics determine the safety control.

For process optimization of the technological parameters, the correct sequence of the sub-processes and the distribution of the sub-processes within an analysis period are important, with a number of side effects such as flushing, rotation, vibration behavior, etc. play a role.

An important factor for the use of the properties of the sub-processes is the time constant with which the processes can determined and the extent to which their result can be traced back to the process via the actuators. The temporal analyzes can already be carried out very quickly today and are quickly incorporated into the control algorithm. Most problems arise when the time constants of the actuators are several powers of ten less than the analyzes. One recognizable solution can be that the determination of critical process states can be predicted even more quickly using model specifications and various partial analyzes.

\section{Bibliography}

[1] Schulze, H.-P. Multiple combination on conventional and non-conventional processes. Intern. Journal of Metal Forming, 2010, 3, pp. 1089-1090.

[2] Wollenberg, G. Schulze, H.-P. Trautmann, K.-H. Kappmeyer, G. Controlled current rise time for pulsed electrochemical machining. Proceedings of ISEM XV, 2007 Pittsburgh, PA, USA pp. 335-338.

[3] Schulze, H.-P. Kröning, O. Herzig, M. Function and formation of PECM-Process-Energy-Sources for specific applications. Proceedings of the ${ }^{14 \text { th }}$ INSECT 2018, Aachen, Germany pp.87-92.

[4] Natsu, K.W. Kurahata, D. Influence of ECM pulse conditions on WC alloy micro pin fabrication. Proceedings of the ISEM XVII Leuven, Belgium. Procedia CIRP 2013, 6 pp. 401-406.

[5] Oßwald, K. Lochmar, I. Schulze, H.-P. Kröning, O. Automated analysis of pulse types in High-Speed Wire-EDM. Proceedings of the ISEM XIX, Barcelona, Spain, 2018 Procedia 68 pp. 796-801.

[6] de Silva, A.K.M. Schulze, H.-P. McGeough, J.A. Zybura, M. Process control and power systems for EC-Erosion Sinking (ELESIN). Proceedings of the ISEM XVI, 2010 Shanghai, China, pp. 389-392.

[7] Schulze, H.-P., Werner, U. Theory of Electro-contact Discharge Machining (ECDM) and its application in Heavy-Duty Processes. Proceedings of the ISEM X 1992, Magdeburg, Germany, pp. 647-653.

PDF automatically generated on 2021-05-23 07:50:44

Article url: https://popups.uliege.be/esaform21/index.php?id=2709

published by ULiège Library in Open Access under the terms and conditions of the CC-BY License

(https://creativecommons.org/licenses/by/4.0) 